resources available through our local, state, and federal health departments. Information is the antidote to terrorism.

Kelly A. Johnson, BA, and Norman Coleman, MD Office of the Assistant Secretary for Preparedness and Response, Department of Health and Human Services

We would like to respond to "Improving Hospital Preparedness for Radiological Terrorism" that was published in the October 2008 issue of Disaster Medicine and Public Health Preparedness.

Through the use of focus group discussions, the authors have identified a critical information gap on the part of our nation's emergency care providers with respect to radiological emergencies. The issues raised in the article highlight the importance of a central source of authoritative information and guidance accessible to providers at the time of an event as well as to those individuals responsible for planning a response to such events. Our goal in writing today is to reach out to your readers as well as the larger community of hospital emergency division providers with important information regarding the Radiation Event Medical Management (REMM) Guidance on Diagnosis and Treatment for Healthcare Providers, a valuable national resource accessible at http://remm.nlm.gov.

REMM was produced by the US Department of Health and Human Services (HHS) in cooperation with HHS' National Library of Medicine and subject matter experts from the other HHS agencies: the National Cancer Institute, the Centers for Disease Control and Prevention, and many United States and international consultants. Its goals are to provide guidance for health care providers about clinical diagnosis and treatment during mass casualty radiological/ nuclear events; provide just-in-time, evidence-based, usable information; and provide Web-based information that is also downloadable in advance to a laptop or personal digital assistant, so that it would be available during an event if the Internet is not accessible.

For medical providers, REMM is a vital source of information relating to radiological and nuclear emergencies. Its resources range from scientific background information and incident scene management to standard operating procedures and patient care guidelines. REMM also provides decision guidelines and templates for use during an actual event.

HHS and its Office of the Assistant Secretary for Preparedness and Response want to assure your readers that the federal government is actively involved in improving our nation's radiological preparedness and response capabilities.

We would highly encourage all interested parties to become familiar with REMM, as well as the many other tools and

\section{MASS CASUALTY TRIAGE: UNIVERSAL VERSUS SPECIFIC}

\section{To the Editor:}

During the last several decades, the incidence of large-scale multicasualty events has significantly increased. Therefore, modern health systems face the new challenge of successfully managing incidents that, on the one hand, involve an unprecedentedly large number of casualties with different types and severities of injury whereas, on the other hand, they are likely to have an unpredictable nature. Thus, I read with great interest the recent article by Lerner et al regarding the standardization of triage methodology across the United States at the time of disaster. ${ }^{1}$ I utterly support the idea that a standard triage method could decrease uncertainty and confusion, by requiring health care delivery teams to follow strict guidelines. This unified approach could be enhanced, however, by the modification of the guideline according to the specific type of mass casualty incident (eg, explosion, shooting, natural disaster). Lessons learned from Israeli and other international experiences in the last few decades have demonstrated that certain types of information-pattern of injuries, number of casualties, and utilization of human medical resources- can be extrapolated to an extent from the information on previous incidents. ${ }^{2}$

Thus, if first responders and other medical teams could get the preliminary information regarding the specific nature of a mass casualty incident, then they would be able to instantly implement a triage protocol tailored specifically to this particular setting. As a result, such compatibility could, presumably, reduce overtriage or undertriage issues, consequently improving patient outcomes. This would likewise help to allocate human medical resources more effectively. For example, according to the Israeli experience, explosions that occurred in buses had the highest rate of overall mortality $(21.2 \%)$ and an unprecedentedly high incidence of head and neck injuries (61.8\%) among survivors, whereas bombing attacks in open spaces resulted in substantially lower overall mortality rates and a high incidence of injuries to extremities $(43.6 \%){ }^{3}$ Given this information, emergency medical services could more accurately plan and implement the prehospital triage protocols. 
In conclusion, the physical setting in which mass casualty events occur dramatically affects the pattern of injury in resulting casualties, their outcomes, and the associated impact on health services. Therefore, this information ought to be integrated with mass casualty triage guidelines.

Oleg Zaslavsky, RN, MHA Doctoral student, University of Washington

DOI: 10.1097/DMP.0b013e31819b0df2

\section{REFERENCES}

1. Lerner EB, Schwartz RB, Coule PL. Mass casualty triage: an evaluation of the data and development of a proposed national guideline. Disaster Med Public Health Preparedness. 2008;2(Suppl 1):S8-S10.

2. Arnold JL, Halpern P, Tsai MC, et al. Mass casualty terrorist bombings: a comparison of outcomes by bombing type. Ann Emerg Med. 2004;43: 263-273.

3. Zaslavsky O. Medical management of mass casualties of suicide bombing: lessons learned from the Israeli experience over the last few years. Paper presented at the annual conference of the Israeli Emergency Nursing Association, Tel Aviv, Israel, March 23, 2003.

\section{Lerner et al reply:}

Thank you for allowing us this opportunity to respond to the thoughtful comments submitted by Mr Zaslavsky regarding our recent article in Disaster Medicine and Public Health Preparedness. Although we agree that the data from Israel are compelling in demonstrating that event circumstances are a factor in predicting injury, our goal was to address the initial sorting of patients by first responders. We believe that we all can agree that the initial responders to a large-scale event will find a scene that is chaotic, and that their first priority will be to bring some type of order to the chaos. The triage process needs to be extremely simple, easy to remember, and rapid; it would not be possible in most circumstances to access a protocol book and determine the best triage process for the circumstances. Furthermore, we designed the SALT triage guideline to be consistent with the move toward an all-hazards approach, and thus the guideline should be applicable for any scenario with multiple patients.
The actual cause of the event may not be clear upon dispatch or even upon arrival of the responders, thereby making it difficult for first responders to use a triage methodology that is alterable based upon etiology. Furthermore, given anecdotal reports of providers responding to mass casualty incidents and not using any consistent triage method, we are concerned that a system that is too complicated will not be used at all.

We would like to be clear that we feel that triage is a dynamic process that typically begins with limited information, resources, and providers. At these initial stages, we believe it would likely be difficult to use a complicated multipossibility triage scheme. As the process unfolds and more information, resources, and providers become available, however, it may be possible for the process to evolve and become more sophisticated, in part by incorporating critical information about the nature of the incident. This may even take the shape of a tool that could be created for hospital personnel to use as casualties arrive. We strongly feel that we need to change the mindset that the initial response to different types of disasters requires a different initial triage approach. The reality is that initially the simplest approach is probably the most likely to be used and therefore to be most effective.

As we state in our article, our goal is to have the SALT triage guideline grow and evolve as the science of triage develops. This guideline should not be viewed as the final product but the beginning of a process that will be driven by science and improved over time. We hope that this is just the beginning of our national dialog on triage, and that we can come to consensus and move forward with a national guideline.

E. Brooke Lerner, PhD, Richard B. Schwartz, MD, Philip L. Coule, MD, Eric S. Weinstein, MD, David C. Cone, MD, Richard C. Hunt, MD, Scott M. Sasser, MD, J. Marc Liu, MD,

Nikiah G. Nudell, NREMT-P, Ian S. Wedmore, MD, Jeffrey Hammond, MD, Eileen M. Bulger, MD, Jeffrey P. Salomone, MD, Teri L. Sanddal, BS, NREMT-P, Graydon C. Lord, MS(c), NREMT-P, David Markenson, MD, and Robert E. O'Connor, MD

DOI: 10.1097/DMP.0b013e3181a4cb70 